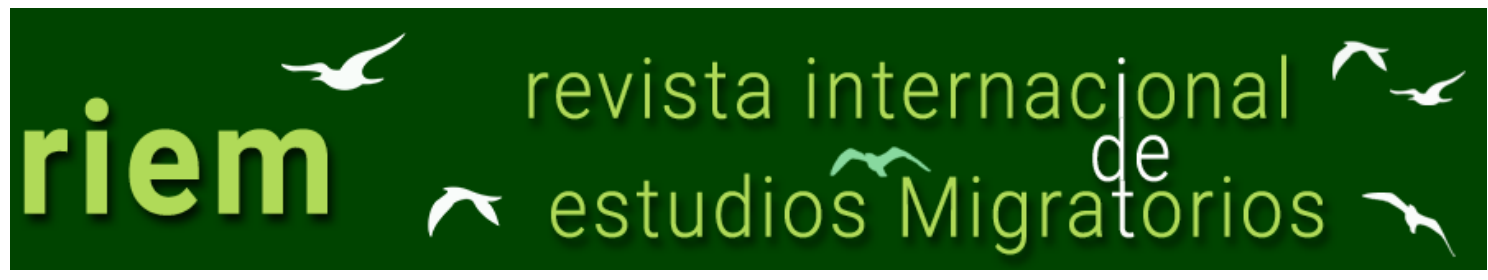

ISSN: $2173-1950$

\title{
Violencia de género en el tráfico de migrantes. Efectos psicosociales y agencia de las mujeres migrantes clandestinas
}

Sius-geng Salinas ${ }^{1}$, Nanette Liberona ${ }^{2}$

Resumen: Los Estados están definiendo políticas migratorias desde una óptica de la seguridad nacional, en la que prima la rigidez de los controles fronterizos y las restricciones en el sistema de visado. Como consecuencia, este tipo de políticas trae consigo el aumento de ingresos clandestinos o cruce de fronteras por pasos no habilitados, fomentando el tráfico de migrantes. El objetivo de este artículo es comprender la experiencia de mujeres migrantes clandestinas que fueron objeto de tráfico, destacando la capacidad de agencia que estas tuvieron a lo largo del trayecto, tráfico y vida en país de destino. Interesa comprender el impacto del tráfico de migrantes como violencia aguda de género, ya que es un eslabón de una cadena de diferentes formas de violencias que viven las mujeres que emigran clandestinamente. La metodología utilizada en esta investigación fue de tipo cualitativa y con un enfoque de género a través del seguimiento etnográfico colaborativo de 6 mujeres migrantes clandestinas residentes en Iquique y Alto Hospicio, región de Tarapacá, Chile, durante el periodo de marzo a agosto de 2018. Los principales resultados en este artículo destacan la presencia de violencia de género transversal a lo largo del trayecto migratorio y en algunas ocasiones previo y desde el inicio del viaje. Destacan las diversas formas de violencia a las cuales se vieron expuestas las mujeres partícipes de esta investigación y los efectos psicosociales

\footnotetext{
${ }^{1}$ Magíster en Relaciones Internacionales y Estudios Transfronterizos, Universidad Arturo Prat, INTE, Iquique, Chile. Sius.sl.p@gmail.com

2 Doctora en Antropología y Sociología, Universidad de Tarapacá, Departamento de Antropología, Iquique, Chile.nliberonac@gmail.com
} 
que esto conlleva, así como la agencia que presentaron. Se concluye que la condición migratoria irregular, propicia una serie de violencias, siendo la expresión de la violencia de género es parte consustancial al tráfico de migrantes y a la vida e inserción en el país de acogida, pues va de la mano con las relaciones patriarcales inconscientes arraigadas en la subjetividad de la sociedad.

Palabras Clave: Tráfico ilícito de migrantes, Violencia de género, Migración femenina, Efectos psicosociales, Agencia. 


\title{
Gender violence in migrant trafficking, psychosocial effects, and agency of clandestine migrant women
}

\begin{abstract}
States are defining migration policies from a national security perspective, in which the rigidity of border controls and restrictions on the visa system prevail. As a consequence, this type of policy brings with it an increase in clandestine income or border crossings through unauthorized crossings, encouraging the smuggling of migrants. The objective of this article is to understand the experience of clandestine migrant women who were trafficked, highlighting the agency capacity that they had throughout the journey, trafficking and life in the country of destination. It is interesting to understand the impact of migrant smuggling as acute gender violence, since it is a link in a chain of different forms of violence experienced by women who emigrate clandestinely. The methodology used in this research was qualitative and with a gender focus through the collaborative ethnographic monitoring of 6 clandestine migrant women residing in Iquique and Alto Hospicio, Tarapacá region, Chile, during the period from March to August 2018. The main results in this article highlight the presence of gender-based violence throughout the migratory journey and on some previous occasions and from the beginning of the journey. They highlight the various forms of violence to which the women participating in this research were exposed and the psychosocial effects that this entails, as well as the agency that was presented. It is concluded that the irregular migratory condition encourages a series of violence, since the expression of gender violence is an inherent part of migrant smuggling and life and insertion in the host country, as it goes hand in hand with unconscious patriarchal relations rooted in the subjectivity of society.
\end{abstract}

Keywords: Smuggling of migrants, Gender violence, Female migration, Psychosocial effects, Agency. 


\section{Introducción}

El fenómeno de la migración es ampliamente estudiado en las ciencias sociales, siendo abordado desde distintas perspectivas y disciplinas. Las migraciones internacionales se caracterizan por ser diversas, y el momento actual del capitalismo las sitúa en una posición de mayor precariedad, al ser soporte de un sistema de acumulación flexible (Ramírez y Álvarez, 2009). El sistema de producción capitalista se fortalece de la migración, irregular, cuya mano de obra es demandada, pero se rechaza a las personas que la proporcionan (Heyman, 2012). Lo que se ve reflejado en la escasa estabilidad y seguridad en el trabajo que tienen las y los inmigrantes, y que puede expresarse principalmente en salarios más bajos que la población no migrante.

En el contexto latinoamericano, nos encontramos frente a una migración diversa, compleja y cambiante: los flujos migratorios ocurren principalmente a escala intrarregional, siendo Argentina, Brasil y Chile los países de destino con mayor número de inmigrantes (Red Jesuita con Migrantes Para América Latina y el Caribe [RJM-LAC], 2018). Si bien el capitalismo fomenta la apertura de las fronteras entre países para promover la circulación de mercancías, se cierra y endurece para el paso de las personas, lo que es planteado como una "paradoja liberal” (Hollifield, 2006). Por consiguiente, los Estados están definiendo políticas migratorias desde una óptica de la seguridad nacional, en la que prima la rigidez de los controles fronterizos y las restricciones en el sistema de visado. Como consecuencia, este tipo de políticas trae consigo el aumento de ingresos clandestinos o cruce de fronteras por pasos no habilitados, fomentando el tráfico y la trata de personas. $\mathrm{Al}$ respecto, "se ha producido una aceleración de la migración en un contexto de control creciente de los migrantes y de refuerzo de las fronteras nacionales y, por consiguiente, una disminución de las posibilidades de una migración "legal" (Bastia, 2009: 78). Ello ha generado que las y los migrantes consideren modos más riesgosos para cruzar las fronteras (Bastia, 2009), contratando el servicio de “coyotes” o "pasadores" para ingresar.

Los feminismos generan aportes relevantes en la academia, en particular en los estudios migratorios se logra la integración del género, permitiendo la comprensión y análisis de las diversas relaciones de género que se encuentran mediando las experiencias migratorias. La implementación de un análisis y metodología feminista reconoce una comprensión total y no fragmentada de la realidad de la migración femenina, prestando atención a cuestiones que por años fueron negadas e invisibilizadas, pero que, gracias a los esfuerzos de diversas feministas, el día de hoy es un tema central para estudiar. Contreras y Trujillo (2017) desde el feminismo decolonial, presentan 3 
puntos para estudiar a la mujer migrante. Para este estudio solo se abordará uno de ellos: la recuperación de la enunciación y experiencia de las mujeres. Lo anterior destaca a las mujeres como protagonistas de sus propias historias, desafiándonos como investigadoras a incluir la dimensión epistemológica de enunciación, es decir, desde donde se investiga. Esto como orientación de una praxis transformativa, que conlleva un dialogo hacia la co-construcción de conocimiento (Contreras y Trujillo, 2017).

Es importante destacar, en base a los estudios existentes, que no todas las mujeres se ven expuestas al mismo tipo de violencias (Rodríguez, 2000), ya que existen diversas cuestiones que se imbrican en la experiencia individual. En el contexto migratorio, una de las conclusiones más relevantes en la literatura es que la condición de género influye en una serie de prácticas discriminatorias, que limitan y disminuyen las oportunidades de desarrollo humano (Hernández y Boix, 2013). De manera tal, las mujeres migrantes están más expuestas a contextos de vulneración de sus derechos (Abril, 2015) que los migrantes hombres, tales como el trabajo forzoso, la explotación sexual, la trata de personas (Hernández y Boix, 2013; Stefoni y Stang, 2017).

Este artículo presenta los principales resultados de nuestra investigación, que tiene como objetivo general comprender la experiencia de mujeres migrantes clandestinas que fueron objeto de tráfico. Esto se vuelve importante por la forma en la que se componen los flujos migratorios, evidenciando las desigualdades de género que determinan las dinámicas migratorias (Mora, 2008). Interesa comprender el impacto del tráfico de migrantes como violencia aguda de género, ya que es un eslabón de una cadena de diferentes formas de violencias que viven las mujeres que migran clandestinamente. Esta expresión aguda de la violencia de género refiere a la intersección de diferentes tipos de violencias que sufren las mujeres a lo largo del trayecto. Por lo tanto, el enfoque de género nos permite analizar la forma en la que se establecen las relaciones de género en la migración y el tráfico, que permite puntualizar las violencias a las cuales se ven expuestas estas mujeres y que muchas veces quedan ocultas. Al mismo tiempo, nos interesa dar a conocer la capacidad de agencia que despliegan las mujeres durante el trayecto y una vez que llegan al país de destino, en este caso Chile. Esto es importante cuando analizamos los efectos psicosociales del tráfico de migrantes; experiencia marcada por violencias que dejan huellas. Para lograr estos objetivos, desarrollamos una investigación basada en una 
metodología cualitativa que se enmarca en un proyecto de investigación mayor ${ }^{3} 4$, el que propone realizar un seguimiento etnográfico colaborativo, con el fin de abordar a esta población.

Este trabajo se enfoca en el trayecto migratorio de seis mujeres migrantes que ingresaron clandestinamente a Chile a través de las fronteras del Norte de Chile con Perú, paso fronterizo Chacalluta (región de Arica y Parinacota), y con Bolivia, paso fronterizo Colchane (región de Tarapacá). El estudio se llevó acabo en la ciudad de Iquique debido al aumento del tráfico de migrantes en los últimos años, vía terrestre, por ambos pasos fronterizos, próximos a la ciudad de Iquique ${ }^{5}$. Tarapacá es además una de las regiones de Chile con mayor proporción de migrantes en relación con su población total, alcanzando un 16,9\% (Servicio Jesuita al Migrante [SJM], 2020).

Este artículo se organiza desde un marco teórico general a uno específico del objeto de estudio, comenzando por el abordaje del binomio género y migración, luego especificamos respecto a la violencia de género y los efectos psicosociales de esta violencia hacia las mujeres migrantes, para terminar con una definición del tráfico ilícito de migrantes. Lo anterior permite comprender los resultados mediante la inclusión de citas textuales de entrevistas, que son inmediatamente analizadas, facilitando la incorporación de la discusión previa a las conclusiones.

\section{Definiciones teóricas y conceptuales a partir del género y la migración}

La relación entre género y migraciones internacionales ha tomado una creciente importancia política y social, como lo evidencian informes de organismos internacionales que se centran en estas problemáticas (Gregorio, 2011). La literatura da cuenta del modo en que se ha desarrollado la línea de investigación sobre migración, mujeres y género, la cual ha contribuido a comprender la construcción de la categoría de migrante como una persona vulnerable y con claras desigualdades estructurales (Stefoni y Stang, 2017). Lo anterior en base a estudios que han demostrado las diferencias salariales, los determinados nichos laborales en los que se insertan, las dificultades en el

\footnotetext{
3 Proyecto Fondecyt de iniciación N. ${ }^{0}$ 11170568, titulado "Ignominia, racismo y agencia en el fenómeno del Tráfico Ilícito de Migrantes que atraviesa las fronteras de Arica y Parinacota y de Tarapacá" dirigido por la Dra. Nanette Liberona Concha.

4 Este artículo es un producto de la tesis de pregrado de la autora principal.

5 Detectan 3.182 ingresos clandestinos de extranjeros a Chile de enero a mayo (El Mostrador, 3 de junio de 2018). 
acceso a la vivienda, entre otros. Si bien esta afirmación es cierta y el contexto al cual se enfrenta la mujer migrante es violento, con una tendencia a la vulnerabilidad, esto no impide que generen diversas estrategias de sobrevivencia y una capacidad de resignificación de sus experiencias. Es importante mantener una mirada equilibrada que evite la victimización de las mujeres, pero a la vez reconocer el contexto adverso al cual se enfrentan y dar a conocer la capacidad de agencia que desarrollaron (Cea y Montenegro, 2014). En este sentido la autora Willers (2016) advierte sobre la migración de mujeres, que este puede ser visualizada tanto desde los procesos de vulnerabilidad como la agencia social, ambos se cruzan con las experiencias que a su vez están mediando las relaciones sociales y psicosociales.

Por procesos de vulnerabilidad entendemos "los efectos del sistema social normativo, simbólico e institucional, que ponen en desventaja a las mujeres indocumentadas" (Willers, 2016: 164). La agencia la comprendemos como la capacidad que tienen las mujeres para resolver las problemáticas a las que se enfrentan, utilizando diferentes estrategias siendo las principales protagonistas de su historia y sus decisiones.

Por otra parte, Tapia (2011) nos advierte que hablar de género en los estudios migratorios no es equiparable a hablar de mujer, lo anterior tiene como consecuencia que el papel del hombre en la migración se ha generalizado y se presta poca atención a cómo los hombres replican las relaciones de género en la migración.

\subsection{Violencia de género}

La violencia hacia la mujer ha adquirido un gran interés por diversos motivos, principalmente la muerte de varias mujeres por sus parejas, como la creciente violencia dentro de las relaciones afectivas. Esto se refleja en la creación de políticas públicas con el fin de prevenir la violencia de género y, en el ámbito académico, en la generación de estudios sobre esta temática.

La violencia de género es definida por la ONU en 1995 como: "Todo acto de violencia sexista que tiene como resultado posible o real un daño físico, sexual o psíquico, incluidas las amenazas, la coerción o la privación arbitraria de libertad, ya sea que ocurra en la vida pública o en la privada”. Bajo esta definición es importante comprender que la violencia de género hacia las mujeres contempla varios tipos de situaciones: socioeconómicas, estructurales, físicas, psicológicas, sexuales, entre otras, que se encuentran profundamente enraizadas y normalizadas en las culturas de todo el mundo, a través de la reproducción de las relaciones de género y la opresión de la mujer, "a tal 
punto que millones de mujeres la consideran un modo de vida” (Acharya y Stevanato, 2005: 2).

Una de las características centrales de la violencia de género es que se ejerce por razones de género, es decir por ser mujer (Hierro, 1998), o por identificarse con una minoría sexual. El punto de encuentro de las diferentes violencias de género es que se efectúan por medio de un acto intencional de poder, ejercido por acción u omisión, con el objetivo de someter, dominar, controlar, e imponer la voluntad de quien la ejerce por sobre la voluntad de la persona que la recibe, transgrediendo derechos y produciendo daños, lo que tiende a ocurrir desde un hombre hacía una mujer (RUVDS, s.f). La violencia de género se expresa en el sistema capitalista patriarcal, que se caracteriza históricamente por relaciones de dominación y opresión establecidas por hombres sobre otros y sobre las mujeres en un sistema económico, político y social que determina la posición de estas en él.

\subsection{Efectos psicosociales de la violencia de género en mujeres migrantes}

Los efectos psicosociales refieren a las áreas que se ven afectadas en la cotidianeidad de una persona y pueden darse tanto a nivel físico, emocional como social. Estos se relacionan directamente con daños que pueden provocar en la salud en general de las personas afectadas. En este apartado tomaremos como referencia el planteamiento de Montañés y Moyano (2006) ya que permite integrar un análisis psicosocial para la comprensión de la violencia de género, teniendo en cuenta factores personales, sociales y culturales. Para los autores, la violencia de género es una problemática considerada multicausal y las mujeres migrantes presentan una dinámica aún más compleja, “debido a la especial vulnerabilidad de este colectivo ya que la violencia ejercida contra las inmigrantes suele ser todavía más encubierta e invisible, debido a las dificultades económicas, sociales y administrativas" (Montañés y Moyano, 2006: 24). Además, en muchas ocasiones la condición de clandestinas las convierte en un colectivo institucionalmente "oculto". Los mismos autores afirman que la violencia de género en población migrante provoca alteraciones emocionales, como el estrés postraumático, inadaptación a la vida cotidiana, malestar emocional, baja autoestima, y destacan la probabilidad de que la violencia de tipo doméstica se intensifique tras la migración.

\subsection{Tráfico ilícito de migrantes}


Los diversos estudios realizados en el ámbito del tráfico ilícito de migrantes (En adelante tráfico) conducen a la conclusión de que la gran mayoría relacionan la trata y el tráfico, lo que dificulta el análisis, impidiendo la claridad y precisión necesaria para abordar el fenómeno (Liberona, 2020). A pesar de las confusiones entre trata y tráfico de migrantes, éstos son delitos diferentes, pero no excluyentes y pueden solaparse. Puede existir en primera instancia tráfico y que posterior a esto se genere la trata de personas, considerada por la Organización Internacional del Trabajo [OIT] como una forma de trabajo forzoso (Instituto Nacional de Derechos Humanos [INDH], 2010).

En cambio, el fenómeno del tráfico ilícito de migrantes es entendido como "una traslación fronteriza de carácter irregular, que de forma particular se encuentra asociada al lucro" (Maldonado, 2010). Asimismo, la víctima de tráfico se define como la persona que viaja voluntaria pero ilegalmente a otro país con la ayuda de terceras partes. Este es el resultado de un acuerdo entre una persona o un grupo/banda que facilita el ingreso a un país por pasos no habilitados; por ende, esquivando los controles fronterizos migratorios (Álvarez, 2007; Hidalgo, 2019; Spener, 2009). Principalmente son los pasadores o "coyotes" que ayudan a llevar a cabo el trayecto que, en algunas ocasiones, contempla el traslado completo, incluyendo el viaje desde el país de origen hasta el de destino (Liberona y Riquelme, 2020). Esta transacción económica, puede costar la vida de la persona que migra, ya que muchas veces el "coyote" extorsiona e incluso pone en peligro a la persona migrante, como lo evidencian los resultados de la investigación de Liberona (2015). Estos demuestran cómo las personas que ingresan a Chile con la ayuda de "coyotes" viven situaciones de extremo despojo de sus pertenencias y también de sus cuerpos. Asimismo, el planteamiento de "la frontera cedazo" (Liberona, 2015) explica que el ingreso por paso no habilitado es resultado de un trato discriminatorio, arbitrario y muchas veces racista ejercido por funcionarios del control migratorio en el ingreso al país. Un trato que conduce a las personas a las que se les niega el ingreso, a cruzar la frontera con la ayuda de "coyotes". En general se concluye que las restricciones al ingreso de extranjeros sólo favorecen el surgimiento de un negocio lucrativo (INDH, 2012).

Desde un análisis feminista del fenómeno del tráfico, lo primero es comprender la frontera como propone Cortés (2018) siguiendo a Bourgois, es decir, un espacio fronterizo que se encuentra atravesado por un continuo de violencia. Este opera a través de 3 mecanismos que son invisibles y la autora señala: 
La violencia estructural (alude al papel de grandes fuerzas políticas y económicas históricamente arraigadas), la violencia simbólica (los sectores de población dominada naturalizan el estatus quo y se culpan por su dominación haciéndola legítima y natural), y la violencia normalizada (refiere a la producción social de la indiferencia ante la brutalidad institucionalizada) (Bourgois, 2010 citado en Cortés, 2018: 41).

Siguiendo el planteamiento de Cortés (2018) nos interesa mantener el foco del análisis en la forma en la que se expresa la violencia a lo largo del trayecto migratorio y en el fenómeno del tráfico, esto permitirá conocer los efectos psicosociales que se contrarrestan con la agencialidad que las mujeres presentaron. Lo anterior es de gran interés ya que como advierte la autora el

Tránsito fronterizo construye a las mujeres extranjeras como víctimas que al proceder de sectores populares son receptoras pasivas de una cultura que les asigna un papel subalterno, olvidando así que ellas desarrollan de manera continua estrategias de subversión y evitación de las imposiciones del sistema (Cortés, 2018: 41).

Lo anterior permite comprender la particularidad que presenta la frontera para el fenómeno estudiado y cómo este se presenta en las experiencias de las mujeres entrevistadas en esta investigación.

\section{Método}

La metodología utilizada en esta investigación fue de tipo cualitativa y con un enfoque de género a través del seguimiento etnográfico colaborativo de 6 mujeres migrantes clandestinas residentes en Iquique y Alto Hospicio, durante el periodo de marzo a agosto de 2018.

Esta metodología considera la dificultad de investigar sobre una población "oculta", como es la migración clandestina. Siguiendo a Meneses (2019), sobre los desafíos de la antropología de la migración clandestina, se diseñó una estrategia de etnografía colaborativa para el levantamiento de la información, realizado de manera progresiva y gradual para establecer relaciones de confianza con las mujeres. Nos inspiramos en la propuesta de "investigación etnográfica activista en colaboración" presentada por Lynn Stephen (2012), quien plantea que "la colaboración produce conversaciones, aportaciones y foros que propician intercambios adicionales" (Stephen, 2012: 232). 
También consideramos lo que plantea París Pombo (2012) respecto a la investigación activista o colaborativa, que nos sitúa en una relación contractual y en un compromiso político con un actor colectivo.

Nuestra metodología consistió, además de la realización de entrevistas y observaciones, en un proceso de acompañamiento jurídico y psicosocial, así como un seguimiento y derivación en el proceso de regularización migratoria y/o de búsqueda del ejercicio de derechos en diferentes instituciones (salud, etc.). Las mujeres fueron contactadas a través de la organización AMPRO (Asamblea Abierta de Migrantes y Pro Migrantes de Tarapacá) y se trabajó a la par con sus dirigentes en los procesos de acompañamiento. El criterio para seleccionar a las mujeres participantes de esta investigación fue que se reconozcan ellas mismas "objeto de tráfico de migrantes", habiendo ingresado a Chile por paso no habilitado con la ayuda de "coyotes". Se realizaron varios encuentros, de los cuales resultaron dos entrevistas en profundidad a cada mujer, llevadas a cabo en sus propias viviendas por la complejidad del tema abordado. Se resguardó la confidencialidad a través de un pseudónimo que ellas mismas eligieron y se aplicó un consentimiento informado a cada entrevistada, aprobado por el Comité de Ética de la Universidad Arturo Prat.

El registro de las entrevistas fue realizado por audio, que fue consentido por las mujeres, posteriormente se realizó la transcripción literal, concluyendo en un análisis por categorías y subcategorías. Sumado además de las observaciones y conversaciones informales con las mujeres entrevistadas que enriquecieron el análisis. Este se realizó por medio del análisis de contenido temático, lo que permitió identificar el significado que las propias mujeres migrantes daban a sus experiencias. Para generar las categorías, se construyeron 3 grandes dimensiones en base a las entrevistas realizadas: decisión de migrar, trayectoria migratoria $\mathrm{y}$ tráfico $\mathrm{y}$ vida en Chile $\mathrm{y}$ que determinan cronológicamente sus vivencias. Las subcategorías emergieron en base a los relatos de las mujeres y, en conjunto a la interpretación de las propias investigadoras, surgen los apartados que serán presentados en los resultados. Para este artículo no se consideró la decisión de migrar ya que este fue desarrollado de manera extensa en la tesis de pregrado de la autora principal. 
Tabla 1. Características de las mujeres participantes

\begin{tabular}{|c|c|c|c|c|}
\hline Nombre & Nacionalidad & $\begin{array}{c}\text { Lugar de } \\
\text { contacto con } \\
\text { coyote } \\
\end{array}$ & $\begin{array}{l}\text { Motivaciones } \\
\text { de migración y } \\
\text { expectativas }\end{array}$ & $\begin{array}{c}\text { Acompañada } \\
\text { en el trayecto } \\
\text { por } \\
\end{array}$ \\
\hline Oloyumiya & Cubana & Guayana & $\begin{array}{l}\text { Trabajar } \\
\text { Situación } \\
\text { económica y } \\
\text { política en país } \\
\text { de origen } \\
\text { Necesidad de } \\
\text { mantener a los } \\
\text { hijos. }\end{array}$ & Sus dos hijos \\
\hline Mayra & Ecuatoriana & Pisiga, Bolvivia & $\begin{array}{l}\text { Reunificación } \\
\text { familiar } \\
\text { Situación } \\
\text { económica en } \\
\text { país de origen } \\
\text { Trabajar } \\
\text { Necesidad de } \\
\text { mantener a los } \\
\text { hijos }\end{array}$ & Sus dos hijas \\
\hline Mery & Cubana & Guyana & $\begin{array}{l}\text { Trabajar } \\
\text { Situación } \\
\text { económica en } \\
\text { país de origen }\end{array}$ & Exesposo \\
\hline Cristina & Dominicana & Ecuador & $\begin{array}{l}\text { Trabajar } \\
\text { Situación } \\
\text { económica en } \\
\text { país de origen }\end{array}$ & Una amiga \\
\hline Katherine & Dominicana & Ecuador & $\begin{array}{l}\text { Trabajar } \\
\text { Situación } \\
\text { económica en } \\
\text { país de origen }\end{array}$ & Una amiga \\
\hline Blanca & Colombiana & Pisiga, Bolivia. & $\begin{array}{l}\text { Trabajar } \\
\text { Situación } \\
\text { económica y } \\
\text { social en país de } \\
\text { origen } \\
\text { Necesidad de } \\
\text { mantener a los } \\
\text { hijos }\end{array}$ & Una amiga \\
\hline
\end{tabular}

Fuente: Elaboración propia.

Las mujeres que participaron son migrantes provenientes de América del sur y el Caribe, que ingresaron al país de manera indocumentada por medio de una transacción económica con "coyotes" o "pasadores", y que viven en la región desde hace 2 año a 5 años. Participaron 6 mujeres, cuyas edades fluctúan entre los 22 y los 52 años, que provenían de Cuba, República Dominicana, Colombia y Ecuador. No hubo una selección con criterios preestablecidos de las participantes, el único requisito era auto reconocerse como objeto de tráfico. Es esta condición la que llevó a estas mujeres a aceptar participar en la investigación, la que además consideraba el acompañamiento antes mencionado, 
lo que resultó atractivo para ellas. El número de participantes fue opcional y relacionado a la capacidad concreta de realizar un adecuado acompañamiento y de procesar la información levantada en el seguimiento etnográfico colaborativo.

\section{Resultados}

Respecto a la organización de los resultados, estos devienen de la información recogida mediante entrevistas y acompañamientos realizados a las mujeres, el orden de los apartados intenta responder a hitos que ocurrieron de manera cronológica. Las categorías utilizadas provienen de la interpretación de las investigadoras en base a la experiencia de las mujeres, con evidencia en su propia voz y experiencia. El primer nivel de análisis refiere a las diferentes expresiones de violencia que sufrieron las mujeres entrevistadas, dando paso al subapartado que aborda el momento en el cual la migración se vuelve tráfico. Luego se aborda desde la perspectiva de género la violencia sexual presente en el trayecto migratorio. En el siguiente subapartado se evidencia lo que Liberona (2015) desarrolla en su investigación: el "rebote" migratorio como condicionante para el tráfico.

Con la intención de visibilizar las pésimas condiciones a las cuales se exponen las mujeres, se crea la categoría de dolor físico y emocional para ser expresado desde su propia vivencia. Como segunda dimensión de análisis, y en concordancia con los planteamientos teóricos, se presentan los efectos psicosociales presentes en los relatos de las entrevistadas. Finalmente, en una tercera dimensión de análisis, y con la intención de no quedar solo en la victimización y denuncia de la situación, se presenta la categoría analítica la agencia. Es importante destacar que todo lo expuesto en estos apartados son situaciones que explícitamente las mujeres pidieron visibilizar.

\subsection{Expresiones de la violencia producida en el tráfico de migrantes}

\subsubsection{Cuando migrar se convierte en "Tráfico"}

De acuerdo el relato de las mujeres que participaron de nuestra investigación, el llamado "tráfico de migrantes", ocurre en diferentes momentos del viaje, es decir al momento de la transacción económica por el servicio, lo cual implica quedar bajo la responsabilidad del "coyote". Las nuevas condiciones sociales y políticas para la migración internacional obligan a las y los migrantes a buscar estrategias como la migración clandestina, con el objetivo de llegar a un destino final y concluir un proyecto migratorio, siendo el tráfico de migrantes una opción para las personas, aun arriesgando 
la vida misma (Bastia, 2009). En algunos casos las mujeres tenían el conocimiento con anterioridad sobre el costo del traslado y sabían a quién acudir. Es el caso de Oloyumiya, de Katherine y de Cristina que desde el inicio de su trayecto toman contacto con un "coyote"; otras solamente al momento del "rebote" en frontera, como sucede con Mayra y Blanca. Cuando el contacto es en el inicio del trayecto, se oferta el traslado completo hasta el lugar de destino, por tanto, se pide un monto grande que incluye el transporte y el hospedaje. En los casos en que el contacto es en el último cruce fronterizo, el "coyote" ofrece sus servicios a quienes son "rebotados" en los controles migratorios. Cada trayecto tiene su particularidad, pero es así cómo se organiza y desarrolla el "tráfico", el que solo se lleva a cabo si es que el pago es inmediato y por el valor que se les pide en el momento, el que oscila entre los $\$ 150$ dólares hasta los \$1000 dólares. En general, este valor varía del inicialmente pactado, como indican los relatos de nuestras entrevistadas. Además, descubrimos una práctica en la que el tráfico de migrantes es forzado, como es el caso de Mery, quien esperaba cruzar la frontera Colchane-Pisiga de manera legal, pero al despertarse se da cuenta que ya está en Chile y es obligada a pagar un monto determinado:

"Nosotros veníamos de Oruro y no sabíamos que cerraban la frontera y en ese bus nos dicen que la frontera abre a las 7 de la mañana, que si queríamos visar el pasaporte teníamos que esperar hasta el otro día y nosotros, era tarde cuando escucho tápense bien que vamos a tener control y era para entrar acá a Chile... antes de llegar a la parada de bus nos pidieron dinero y tuvimos que pagarles. La idea era visar, que era la mejor forma de ingresar y no...cuando llegamos acá tuvimos que pagar como 500 dólares cada uno" (Mery, Iquique, mayo 2018).

Por otra parte, los relatos de las mujeres dan cuenta de la relación con el o los “coyotes" que está marcada por el abuso de poder a través de cobros exagerados, la subalternidad y el robo. Asimismo, en un caso pudimos apreciar la entrega de una falsa "visa", con la cual supuestamente se podía trabajar y permanecer legalmente en Chile:

"Prácticamente el señor que nos trajo de Tacna a Bolivia nos dijo 'las llevamos a Iquique y allá ustedes van a tener trabajo', incluso el me dio un papel firmado y sellado que ese era un papel de entrada con la visa y que con eso a mí no me molestaban acá, que yo estaba legal, pero cuando yo entré aquí me di cuenta que no era cierto, que eso era falso... que yo podía caer presa con eso, en realidad era víctima pero fui participe de esa cosa, porque yo lo acepté, pero igual a veces la ignorancia hace muchas cosas, 
pero incluso yo lo llevé a la pastoral y ella me dijo porque no lo llevé a la PDI y dije que fui engañada, pero me dio miedo, me dio cosa y no lo quise hacer" (Cristina, Iquique, agosto 2018).

Este trayecto se caracteriza por el poder que ejercen los "coyotes" sobre las personas, las que son altamente vulnerables tanto por las condiciones físicas, la clandestinidad, como por el sentir subjetivo de las mismas, que está marcado por el miedo:

"Ellos comenzaron a revisar las maletas y dijeron: 'es que tenemos que revisar por si ustedes llevan drogas', después tenemos problemas nosotros y así agarró y empezó. Entonces había alguien que tenía muchos productos de belleza: gel, bálsamo, esas cosas que él había traído para una familia de él, que estaba aquí, que tienen una peluquería, y le quitaron la maleta completa” (Katherine, Iquique, agosto 2018).

"Incluso yo no quería pagar y me decían: 'tienes que pagar porque yo pasé a unos cubanos y me pagaron 1000 dólares'. Ay, el señor era prepotente, él quería su dinero...el señor estaba como alterándose... a mí me intimidada, bueno finalmente le dimos 900 dólares que era lo que teníamos" (Mery, Iquique, mayo 2018).

\subsubsection{La presencia de violencia sexual}

Es así como a lo largo del trayecto y por medio del relato de las mujeres, se destaca la presencia de violencia sexual. El espacio migratorio fronterizo se encuentra atravesado y construido sobre las relaciones de poder, contribuye y sustenta el orden de género contextualizando las relaciones de poder entre mujeres y hombres (Cortes, 2018). En esta relación de dominación en base al género a la que se ven sometidas, se observan diferentes formas de violencia. En cuanto a la violencia sexual, se identifica su presencia de dos maneras: en tanto intento de agresión sexual y como acoso sexual, así como tocamientos de carácter sexual. Cabe mencionar que, durante el trayecto, no solo los "coyotes" son identificados por las mujeres como perpetradores de violencia sexual, sino también agentes policiales. En su relato, Katherine narra que los "coyotes" intentan mantener relaciones sexuales con las mujeres que transportaban, además de verbalizar insinuaciones de carácter sexual:

"Entonces cuando estuvimos ahí ellos llegaron en la noche y empezaron a decir que para qué íbamos para Chile, si es que íbamos a prostituirnos con eso fue que llegaron los coyotes...eran todos bolivianos 
y decían 'van a singar', esa era la palabra que usaban, 'singar' es como prostituirse y tener relaciones aquí y nosotras le decíamos 'no, y porqué usted nos falta el respeto de esa forma', estaban medios borrachos y nos decían que nos pagaban para acostarnos con ellos" (Katherine, Iquique, agosto 2018).

Así, se evidencia una clara determinación de las relaciones de género en contexto migratorio, y de la violencia de género que se reproduce, específicamente en el contexto del tráfico de migrantes. Finalmente, esta forma de migrar da paso a la clandestinidad, por lo tanto, a pesar de que todos los y las migrantes clandestinos/as corren peligro de sufrir diferentes tipos de vulneraciones, cuando esa persona es una mujer, tiene más probabilidades de sufrir mayores abusos, diferentes tipos de violencia y discriminaciones (Acharya y Stevanato, 2005).

Por otra parte, el miedo de ser violada a lo largo del trayecto se encuentra presente en el relato de Cristina, quien agradece el no recibir un ataque sexual. En este sentido, Cortés (2018) señala:

"El miedo a la violación genera prácticas de confinamiento y de control del movimiento de las mujeres. El género es un principio central en la organización de la movilidad de las mujeres, y la violencia sexual y de género modela las experiencias y las percepciones de las mujeres al basarse en la existencia de desequilibrios de poder en determinados contextos sociales y culturales, formas de control interpersonales, posiciones de desventaja social frente a los hombres, pautas de construcción y orientación de la identidad, y se caracteriza por su invisibilidad, normalidad e impunidad" (Cortés, 2018: 46).

Así apreciamos la forma en la cual el género y la violencia sexual determina el trayecto migratorio y sobre todo el tráfico, siendo una carga emocional importante para Cristina el poder sufrir un ataque de estos.

\subsubsection{El fenómeno del rebote como condicionante para la clandestinidad migratoria}

En el afán de comprender las razones por las cuales ocurre el fenómeno de tráfico de migrantes a través de las experiencias de las propias mujeres e incorporando la noción de "frontera cedazo" planteada por Liberona (2015), se desprende el fenómeno de "rebote" fronterizo. Al existir el "rebote" -la negación del ingreso al país-, el ingreso clandestino y el tráfico pasa a ser una opción (Liberona, 2015). 
"En Tacna nos fuimos en taxi que les dicen acá, fuimos a la frontera y no nos dejaron pasar, porque nos dijeron que teníamos que tener una 'bolsa de viaje' de 700 dólares o 1000 dólares y nos regresaron [...] Ella es morena, bien morena, quien me acompañaba mi amiga, yo creo que eso influyó, porque la vieron a ella y dijo: 'no, no, se devuelven y no pasan'... nos marcaron los pasaportes y nos devolvieron en un bus para Tacna" (Blanca, Iquique, agosto 2018).

"No pude ingresar y ahí se fregó todo, cuando ingresaron, solo entraron las niñas y él [marido], y yo me quedé ahí en Colchane, me quedé sola prácticamente, la pasé como una semana y media por allá sola, la pasé muy mal porque no tenía ni plata” (Mayra, Iquique, abril 2018).

\subsubsection{Dolor físico y emocional}

Las condiciones geográficas y físicas del ingreso a Chile por Chacalluta o por Colchane son bastante adversas y en general este se realiza a pie. En el primer caso, el cruce es por el desierto costero, en un sector en el que se encuentran minas antipersonales dispersas, por lo que no hay que perderse de la ruta indicada por los "coyotes", siguiendo la línea del tren. En el segundo caso, la dificultad radica en el clima extremo del desierto altiplánico. Los cruces son principalmente de noche, momento en que se presenta el frío desértico acompañado de vientos que dificultan la caminata. Las experiencias de las mujeres durante el trayecto hasta llegar a Chile están atravesadas por mucho dolor corporal y emocional por lo que tuvieron que atravesar. Este dolor se sigue expresando en su cotidianeidad, muchas de ellas refieren aún no superar lo ocurrido, por lo cual, se torna importante la comprensión de los efectos psicosociales relacionados con el fenómeno.

"Yo tenía los pies hinchados por tanto trayecto, porque fue mucho lo que duramos en un bus, tu durabas hasta 36 horas y así, fue terrible, ya no quería más" (Katherine, Iquique, agosto 2018).

"Hubo momentos que pensamos mejor volver a casa, pero yo le decía a la compañera que no, que vamos a seguir hacia delante que Dios está con nosotros" (Cristina, Iquique, agosto 2018).

Además, durante el tráfico una de las mujeres relata que fue secuestrada y encerrada en contra de su voluntad por los "coyotes", una vez en Chile. Además, fue abandonada por estos mismos en medio del desierto y en condiciones precarias. Al regresar, estas 
personas comenzaron a hacer insinuaciones de tipo sexual bajo el efecto del alcohol, lo que da cuenta de la exposición a la cual se enfrentan las personas al utilizar este tipo de servicio. En síntesis, existió 1) Robos y abusos económicos en los cobros por el servicio que entregan; 2) Exposición a riesgos y abandono por parte de los coyotes durante el trayecto; 3) Violencia sexual de diversa índole.

\subsection{Efectos psicosociales y económicos del cruce clandestino}

Los factores subjetivos son importantes en la configuración de las experiencias de violencia de las mujeres entrevistadas, en tanto consecuencias psicosociales de la vivencia de violencia de género (Montañés y Moyano, 2006). Los malestares que hasta el día de hoy significan respecto a sus vivencias a lo largo del trayecto migratorio refieren a sentimientos de mucho miedo y ganas de no querer recordar las situaciones violentas por las que tuvieron que pasar. Estos lamentos son relatados por la mayoría de las mujeres, acompañados de sentimientos de mucho dolor, junto a llanto al narrar la violencia y amenazas que tuvieron que vivir. Una de ellas al haber experimentado este trayecto clandestino expresa agradecimiento de no haber sido violada en el camino. Ello da cuenta de lo inminente que se encuentra el riesgo de sufrir violencia sexual en el trayecto migratorio, lo que reafirma la presencia de relaciones patriarcales y de género en los procesos migratorios (Bastia, 2009; Tapia, 2010).

En este contexto, la violencia de género se expresa como una importante amenaza al bienestar psicosocial, que podemos distinguir en: 1) Violencia doméstica, que se expresa principalmente de manera psicológica, 2) Infidelidad por parte de la pareja y 3) Abandono de la pareja por embarazo no planificado.

En cuanto a la violencia doméstica, podemos mencionar el caso de Mayra, quien se enfrenta constantemente a diferentes tipos de manipulación por parte de su pareja. Debido a su situación migratoria, su pareja la manipula emocionalmente diciéndole que si sale a la calle la encontrará la PDI (policía) y será expulsada. Esta no es la única manipulación; luego de una infidelidad por parte de él, amenaza a Mayra sutilmente sobre su situación migratoria, insinuando la posibilidad de separarse, ejerciendo principalmente presión respecto a que ella no se puede ir con sus hijas e hijo (3), ya que es él quien tiene la tuición de las niñas y el niño ${ }^{6}$. Esto da cuenta de una manera en

\footnotetext{
${ }^{6}$ Cuando Mayra es rebotada en la frontera iba con sus dos hijas. Su marido, quien ya tenía regularizada su situación migratoria en Chile, cruza la frontera desde Chile para volver a ingresar con las hijas, obteniendo de esta manera la tuición de ellas.
} 
particular a la cual las mujeres se ven expuestas a la violencia de género por su situación migratoria. Es aquí donde las relaciones de género tienen un rol fundamental en el desarrollo de las migraciones (Bastia, 2009), afectando a las mujeres de diversas formas.

Otro caso es el de Mery, quien después de unos meses en Iquique junto a su pareja con la que migró, es abandonada por él cuando este se entera del embarazo.

"Bueno yo estaba a punto de volverme loca, yo no sabía si regresarme pa' Brasil, si sacar pasaje pa' Cuba, si quedarme aquí, ya no sabía qué hacer, porque igual sin papeles no me querían dar trabajo, ni en tienda, ni en ningún lado. Yo estuve caminando por el centro, por Vivar y me estaban pidiendo papeles, para trabajar de vendedora, y antecedentes penales. Y yo me hallaba aquí sin salida, porque no tenía trabajo, no tenía papeles, no tenía dinero, no tenía nada” (Mery, Iquique, mayo 2018).

La condición migratoria que conlleva una especificidad en la expresión de la violencia de género en las mujeres trae a su vez una serie de irregularidades, engaños y vulneración de derechos, principalmente al acceder a algún trabajo. Sin embargo, esto no es excluyente a otros ámbitos de la vida de las mujeres.

"Yo salía a trabajar, trabajé una vez con una señora en el agro, dos meses y no me pagó, y mi deuda iba corriendo y corriendo allá en Dominicana, los intereses [...] También trabajé con una peruana en su casa haciendo aseo y tampoco me pagó, me pagó, pero a plazo me daba 50, después 70... a mí no me servía para mandar para allá, esos fueron los primeros tres meses que yo trabajé aquí, no pude hacer nada” (Katherine, Iquique, agosto 2018).

"Las señoras se aprovechaban de eso, de no tener mis papeles y me decían espérate aquel día y nada y así me tenían y no me pagaban” (Mayra, Iquique, abril 2018).

"Trabajé en un restaurant, uno peruano, unas 3 semanas... no volví a trabajar más porque tuve problemas con la dueña, era muy exigente, se aprovechaba por no tener papeles” (Mery, Iquique, mayo 2018).

Asimismo, el quedar sin dinero al finalizar el trayecto es una característica que comparten todas las mujeres participantes de esta investigación y que resolvieron de diferentes formas, tal como lo expresan sus relatos: 
"Luego de pagar los \$900 dólares quedamos sin dinero" (Mery, Iquique, mayo 2018).

“Al final me quedaban como \$70 y los gasté el primer día que llegué para pagar una habitación y no tenía ni para comer" (Katherine, Iquique, agosto 2018).

"Cuando llegué a Iquique no tenía nada de plata, así que me fui a la pastoral migrante, fue terrible de verdad que si" (Cristina, Iquique, agosto 2018).

"La pasé muy mal, porque no tenía ni plata para pagar el coyote, fue con mi marido al llegar a Iquique que le pagué" (Mayra, Iquique, abril 2018).

El fenómeno del tráfico trae consigo la clandestinidad, la cual está marcada por el acceso a trabajos precarizados, diversas dificultades para el acceso a la salud, vivienda y principalmente la regularización de la situación migratoria. Situación que está mediada por el miedo, la soledad, la desesperación de no saber qué hacer y cómo encontrar un trabajo, lo que afecta el bienestar psicosocial de las mujeres.

Sobre las aspiraciones laborales de las mujeres migrantes entrevistadas, estas señalan querer trabajar en lo que encuentren, ya que necesitan el dinero para vivir. Se dirigen a las ofertas de trabajo disponibles, las que conocen mediante las redes que van estableciendo. Respecto a los trabajos que acceden en Iquique, la principal característica de estos es que son realizados de manera informal, es decir que no existe una relación contractual de los empleadores con las mujeres. La informalidad es el primer paso para la precarización laboral, ya que no existe obligación de cumplir con los derechos laborales y los pagos. Las mujeres se vieron expuestas a esta vulneración que trajo consigo un gran costo en todo ámbito de su vida.

\subsection{Expresiones de la capacidad de agencia para enfrentar la violencia de género}

Los relatos de las mujeres dan cuenta de las diferentes experiencias que atravesaron a lo largo del trayecto hasta llegar a la ciudad de Iquique. Si bien existen características similares, cada historia es diferente y presenta sus particularidades. Las decisiones que toman reflejan las diversas estrategias que implementaron a fin de resolver los conflictos que se les presentaban, con el propósito principal de concretar su proyecto migratorio. 


\subsubsection{Estrategias económicas}

Una de las problemáticas que deben resolver en el trayecto es el cálculo económico que realizan antes y durante el viaje. De esta manera desplegaron estrategias económicas, por ejemplo, algunas viajan con el valor exacto que les fue indicado para el pago del servicio del "coyote". Otras viajan con una cantidad de dinero específica y, al quedar sin dinero, trabajan en el país de tránsito en el que se encuentren para poder continuar el viaje. Algunas piden dinero a sus familiares para poder continuar cuando los cobros sobrepasan lo acordado, también hipotecan y venden propiedades a fin de llegar al destino final, adquiriendo deudas, ya que regresar al país de origen no es una opción, como lo demuestran los siguientes extractos de entrevistas:

"Nosotros teníamos que venir con mil dólares, que eran lo que pedían supuestamente para el viaje como 'bolsa de viaje', porque vienes como turista. Yo fui con los mil dólares justos, porque tampoco podía hipotecar la casa de mi abuelo por más, porque ya iba a ser mucho el gasto, porque yo lo cogí con intereses con $12 \%$... era mucho, yo solo de intereses tenía que pagar \$200.000 pesos chilenos” (Katherine, Iquique, agosto 2018).

"[a mitad del trayecto] Yo hablé con mi familia para que me mandara el dinero y llegar acá, le dije al papá de mis hijas que tengo un solar, que lo hipotecara y me mandara ese dinero, qué más puedo hacer...un terreno agrícola, lo hipotecó y me mandó el dinero, ellos recibieron el dinero, porque lo mandaron a nombre de ellos, ni siquiera vi el dinero" (Cristina, Iquique, agosto 2018).

"Ya no tenía más plata, así que tuve que trabajar en Perú una semana, y después en Tacna una semana más” (Oloyumiya, Iquique, mayo 2018).

Apreciamos diversas estrategias para sortear una misma situación, siendo relevantes sus familias y las deudas adquiridas que posterior al viaje deben comenzar a pagar.

\subsubsection{Redes de apoyo}

Se evidencia además que la migración no es vivenciada de manera solitaria; en el caso de Blanca, Katherine y Cristina migran acompañadas de amigas, lo que da cuenta de la importancia del respaldo existente entre mujeres (Pacecca, 2007) y las estrategias que se generan se podrían comprender de cuidado colectivo. 
"Yo me vine con una amiga de mi mamá a Chile, para no venirme sola, ella es mayor, pero nos vinimos juntas" (Katherine, Iquique, agosto 2018).

"Yo viajé en compañía de una prima mía, veníamos las dos, así que pasamos todo juntas, pero iay! que terrible, no quiero ni recordar todo lo que pasamos" (Cristina, Iquique, agosto 2018).

Además, en los relatos de las mujeres se identifica una alta solidaridad entre migrantes quienes intentan apoyarse constantemente. Este elemento es muy relevante para que las mujeres puedan, desde su agencia, insertarse en la sociedad, con otras migrantes y con el resto de la sociedad chilena. Algunas de estas mujeres participan en organizaciones sociales sin fines de lucro, como la Asociación de dominicanos y AMPRO (Asamblea Abierta de Migrantes y Pro Migrantes). En cuanto a AMPRO, se ha generado una comunidad de migrantes y pro migrantes7, liderada por mujeres migrantes, en la cual existe apoyo mutuo y una orientación en aspectos jurídicos y sociales que aportan a la resolución de la situación migratoria irregular. También existe un apoyo afectivo y orientación para la inserción laboral o acceso a salud, mas, nada en específico respecto a las situaciones de violencia de género vividas por las mujeres migrantes. Si bien este punto es muy importante, no se profundizará en este texto y se espera que trabajos posteriores den cuenta en detalle de las redes existentes.

\section{Conclusiones}

La condición migratoria irregular propicia una serie de violencias. No obstante, el comprender la violencia de género como determinante estructural, permite problematizar la especificidad que conlleva el ser mujer, migrante y clandestina. Esto puede apreciarse en la particularidad de cómo se expresa y se vivencia dicha violencia en contextos microsociales. Concluimos que la expresión de la violencia de género es parte consustancial al tráfico de migrantes y a la vida e inserción en el país de acogida, pues va de la mano de las relaciones patriarcales inconscientes arraigadas en la subjetividad de la sociedad.

Es importante comprender que el trayecto migratorio, el tráfico de migrantes y la inserción en el país receptor, en este caso Chile, están íntimamente vinculados entre sí,

\footnotetext{
7 Los miembros de AMPRO se identifican con la defensa de los derechos de las personas migrantes, por tanto, pueden ser inmigrantes regulares o irregulares de cualquier nacionalidad y personas chilenas.
} 
ya que forman parte de la historia y experiencia de cada una de las mujeres que participaron en esta investigación, cada una con sus particularidades. En un primer acercamiento, es importante problematizar la criminalización constante que se genera frente a la persona migrante en la sociedad chilena, lo que se expresa claramente en el "rebote" en la frontera. En relación con lo anterior, y debido a que el tráfico de migrantes está tipificado como un delito, muchas veces ocurre una visión criminalizadora de las personas que han ingresado de manera clandestina al país, lo que conlleva desigualdades importantes en el país de destino. Por eso el interés de esta investigación en destacar y nombrar la experiencia como "migración clandestina", pero no perdiendo de vista la especificidad del fenómeno del tráfico para comprender lo que deben enfrentar las mujeres al elegirlo opción de su migración. La elección de aquello se encuentra mediada por las políticas migratorias restrictivas, el rebote fronterizo, la reunificación familiar, las expectativas de vida en Chile y el no tener dinero suficiente para regresar a su país de origen en el caso de que el proyecto migratorio se haya visto truncado.

La contribución fundamental de este trabajo consiste en poner de manifiesto la violencia de género del trayecto migratorio de las mujeres traficadas, así como su fortaleza a pesar de las circunstancias que atraviesan, Es así como el tráfico constituye un eslabón más dentro de la configuración de la violencia cotidiana que deben lidiar las mujeres. Por eso, el interés en comprender la situación de la mujer migrante clandestina debe ser mayor, debido a que -como ha demostrado este estudio-, las expresiones de aquella son específicas e invisibilizadas para esta población. Sin embargo, a pesar de existir un contexto altamente vulnerable para ellas, no les impide generar estrategias constantemente, como trabajar por cuenta propia, buscando redes principalmente de mujeres, con las cuales se organizan y apoyan. Por lo cual, se torna importante la capacidad que presentan para ir construyendo y resignificando sus experiencias, no quedándose en situación de víctimas como tal, sino de protagonistas de sus vidas.

Por lo tanto, a través de sus relatos pudimos conocer el dolor que esta experiencia significó en sus vidas y los efectos psicosociales asociados a sus propias trayectorias migratorias, y que para algunas aún afecta en la actualidad. Los efectos psicosociales ligado a lo anterior se vio reflejado en diferentes ámbitos de la vida de las mujeres, que pudimos poner de manifiesto a través de sus relatos como por ejemplo la reducción en la calidad de vida. Lo anterior contribuye a efectos secundarios tanto para la familia o seres cercanos, presentando alteraciones emocionales generalizado con dificultades para la realización de diversas actividades cotidianas. 
Es importante señalar que dada la complejidad de la población objeto de investigación, nos encontramos con ciertas limitaciones, que se deben considerar en futuros estudios referidos a esta problemática. Por un lado, las mujeres no siempre estuvieron disponibles para el acompañamiento, incluso cuando lo que se proponía realizar iba en su beneficio. Generalmente era difícil contactarlas por teléfono y había que ir a sus viviendas para saber cómo se encontraban. Asimismo, su condición de alta precariedad limita sus posibilidades de estar conectadas a internet, lo que en varias ocasiones significó tener que reagendar un encuentro. Por otro lado, la condición de irregularidad administrativa implica un temor permanente a ser deportadas, esto genera relaciones complejas, en las que es difícil disociar la colaboración para el ejercicio de derechos con el asistencialismo.

Por último, afirmar que las relaciones de género y en muchos casos la violencia asociada a ellas interfiere de manera transversal en el fenómeno del tráfico ilícito de migrantes y también país de destino. Aquí la importancia de reconocer la forma en la cual se configuran las relaciones de poder en el sistema capitalista patriarcal, que pone en desventaja a las mujeres. De esta manera es posible comprender la posición inferior que ocupa la mujer migrante en de la sociedad capitalista, sin negar la capacidad de agencia que las mujeres han presentado históricamente y en especial en los relatos que pudimos escuchar y analizar en este trabajo.

\section{Referencias}

Abril, M. I. (2015). La interpretación en contextos de violencia de género con referencia al caso español. Revista de traductología, 19(1), 77-94. https://doi.org/10.24310/TRANS.2015.v1i19.2093

Acharya, A. y Stevanato, A. (2005). Violencia y tráfico de mujeres en México: una perspectiva de género. Revista Estudios Feministas, 13(3), 507-524. https://dx.doi.org/10.1590/S0104-026X2005000300003

Bastia, T. (2009). La feminización de la migración transnacional y su potencial emancipatorio. Papeles de relaciones ecosociales y cambio global, 104, 67-77.

Cea, P. y Montenegro, M. (2014). Más allá de la visibilización: problematizando discursos sobre violencia de género en la pareja contra mujeres inmigradas en España. $\begin{array}{llll}\text { Quadernos de } & \text { Psicología, 16(1), }\end{array}$ https://doi.org/10.5565/rev/qpsicologia.1194 
Contreras, P. y Trujillo, M. (2017). Desde las epistemologías feministas a los feminismos decoloniales: a portes a los estudios sobre migraciones. Athenea Digital Revista de Pensamiento e Investigación Social, 17(1), 145-16.

Cortés, A. (2018). Violencia de género y frontera: migrantes centroamericanas en México hacia los EE. UU. European Review of Latin American and Caribbean Studies, 105, 39-60. https://doi.org/10.18352/erlacs.10321

El Mostrador. (3 de junio de 2018). Detectan 3.182 ingresos clandestinos de extranjeros a Chile de enero a mayo. El Mostrador. https://m.elmostrador.cl/noticias/2018/06/03/detectan-3-182-ingresosclandestinos-de-extranjeros-a-chile-de-enero-a-mayo/

Gregorio Gil, C. (2011). Análisis de las migraciones transnacionales en el contexto español, revisitando la categoría de género desde una perspectiva etnográfica y feminista. Nueva Antropología, 24(74), 39-71.

Hernández, I. y Boix, A. (2013). Haciendo camino al andar: migración, feminización y trata de personas en los flujos de migración irregular de la frontera sur de México. Revista Digital Universitaria, 14(7).

Heyman, J. (2012). "Capitalismo, movilidad desigual y la gobernanza de la frontera México- Estados Unidos, Desafiando fronteras”. En A. Aquino, A. Varela y F. Décosse (Eds.), Control de la movilidad y experiencias migratorias en el contexto capitalista (pp. 25-40). Oaxaca.

Hidalgo. (2019). La producción de la irregularidad en la condición migratoria. Un estudio sobre el ejercicio de los derechos humanos de las personas irregularizadas en Ecuador 2008-2018. (Tesis de magister). Universidad Andina San Simon Bolivar, Ecuador, Quito.

Hierro, G. (1998). La violencia de género. En V.A. Sánchez, (Ed.), El mundo de la violencia (pp. 263-273). México. Universidad Nacional Autónoma de México y Fondo de Cultura Económica.

Hollifield, J. (2006). El emergente Estado migratorio. En A. Portes y J. Dewind (Eds.), Repensando las migraciones (pp. 67-96). México: Miguel Ángel Porrúa, Universidad Autónoma de Zacatecas, Secretaría de Gobernación. Instituto Nacional de Migración.

Instituto Nacional de Derechos Humanos, (INDH). (2010). Informe Anual: Situación de los Derechos Humanos en Chile. 
Instituto Nacional de Derechos Humanos, (INDH). (2012). Informe Anual: Situación de los Derechos Humanos en Chile.

Liberona, N. (2015). La frontera como cedazo y el desierto como aliado. Prácticas institucionales racistas en el ingreso a Chile. Polis, 42, 20-37.

Liberona, N. (2020). El rol de las fronteras en el fenómeno del trafico de migrantes. En H. Dilla y F. Neira (Eds.), Hacia una teoría de las fronteras latinoamericanas. INTE-UNAP/CIALC-UNAM. Manuscrito presentado para su publicación.

Liberona, N. y Riquelme, D. (2020). Una propuesta metodológica corporal para el estudio de la migración clandestina desde la antropología. Religación. Revista De Ciencias Sociales $Y \quad$ Humanidades, 5(24), 103-116. https://doi.org/10.46652/rgn.v5i24.655

Meneses, G. A. (2019). La antropología de las migraciones clandestinas en tiempos de neo-movilidades alternativas y el muro de Donald Trump. Religación. Revista de Ciencias Sociales y Humanidades, 4(13), 16-31.

Montañés, P. y Moyano, M. (2006). Violencia de género sobre inmigrantes en España. Un análisis psicosocial. Pensamiento Psicológico, 6, 21-32.

Mora, C. (2008). Globalización, Género y Migraciones. Polis, 20, 285-297. https://doi.org/10.4067/s0718-65682008000100015

Pacecca, M. I. (2007). Género y trayectoria migratoria: mujeres migrantes y trabajo doméstico en el AMBA. IX Jornadas Argentinas de Estudios de Población. Asociación de Estudios de Población de la Argentina. Huerta Grande, Córdoba.

Pombo, P. (2012). De la observación participativa a la investigación militante en las ciencias sociales. El estudio de las comunidades indígenas migrantes. En M. Ariza y L. Velasco Ortiz (Eds.), Métodos cualitativos y su aplicación empírica. Por los caminos de la investigación sobre migración internacional (pp. 241-270). UNAM, Colegio de la Frontera Norte, México.

Ramírez, J. y Álvarez, S. (2009). “Cruzando Fronteras”: una aproximación etnográfica a la migración clandestina ecuatoriana en tránsito hacia Estados Unidos. Confluenze. Rivista di Studi Iberoamericani, 1(1), 89-113. https://doi.org/10.6092/issn.2036-0967/1423

Red Jesuita con Migrantes Para América Latina y el Caribe (RJM-LAC). (2018). Desafíos de los flujos migratorios desde la perspectiva de la Red Jesuita con Migrantes para América Latina y el Caribe. 
Red Uruguaya Contra la Violencia Doméstica y Sexual, RUVDS (s.f) .Violencia basada en género. Disponible en: https://www.violenciadomestica.org.uy/publicaciones/Lib1\%20Violencia\%20de \%20genero-L.pdf

Rodríguez, M. (2000). Intersecciones de raza, clase y género en Nuevo México. Política y Cultura, 14, 109-131.

Servicio Jesuita a Migrantes, (SJM). (2020). Migración en Chile. Anuario 2019, un análisis multisectorial. Santiago, Chile. Disponible en https://www.migracionenchile.cl/publicaciones

Spener, D. (2008). El apartheid global, el coyotaje y el discurso de la migración clandestina: distinciones entre violencia personal, estructural y cultural. Migración y desarrollo, 10, 127-156.

Stefoni, C. y Stang, F. (2017). La construcción del campo de estudio de las migraciones en Chile: notas de un ejercicio reflexivo y autocrítico. Revista Íconos, 58, 109-129.

Stephen, L. (2012). Investigación en colaboración y su aplicación a la investigación de género en organizaciones transfronterizas. M. Ariza y L. Velasco (Eds), Metodología cualitativa y su aplicación empírica, por los caminos de la investigación sobre migración internacional (pp. 187-240). UNAM, Colegio de la Frontera Norte, México.

Tapia, M. (2010). Inmigración boliviana en España: Un caso para la comprensión de la migración internacional con perspectiva de género. Tinkazos, 28, 109-127.

Tapia, M. (2011). Género y migración: trayectorias investigativas en Iberoamérica. Encrucijada Americana, 4(2), 115-147.

Willers, S. (2016). Migración y violencia: las experiencias de mujeres migrantes centroamericanas en tránsito por México. Sociológica, 31(89), 163-195. 